(C) 2011 IEEE. Personal use of this material is permitted. Permission from IEEE must be obtained for all other uses, in any current or future media, including reprinting/republishing this material for advertising or promotional purposes, creating new collective works, for resale or redistribution to servers or lists, or reuse of any copyrighted component of this work in other works. 


\title{
FamTV: An Architecture for Presence-Aware Personalized Television
}

\author{
Patricia Arias Cabarcos, Member, IEEE, Rosa Sánchez Guerrero, Member, IEEE, \\ Florina Almenárez Mendoza, Member, IEEE, Daniel Díaz-Sánchez, Member, IEEE, \\ and Andrés Marín López, Member, IEEE
}

\begin{abstract}
Since the advent of the digital era, the traditional TV scenario has rapidly evolved towards an ecosystem comprised of a myriad of services, applications, channels, and contents. As a direct consequence, the amount of available information and configuration options targeted at today's end consumers have become unmanageable. Thus, personalization and usability emerge as indispensable elements to improve our content-overloaded digital homes. With these requirements in mind, we present a way to combine content adaptation paradigms together with presence detection in order to allow a seamless and personalized entertainment experience when watching $T V^{I}$.
\end{abstract}

Index Terms - Digital TV, content personalization, identity management, presence detection.

\section{INTRODUCTION}

Convergence has influenced consumer electronic devices, especially those capable of displaying TV, and content distribution, leading to a revolution in the television market. In fact, the combination of traditional broadcasting TV services and video streaming, either over Telco networks or the Internet, sets up the trend. Emerging standards such as HbbTV [1] promote the utilization of hybrid set-top boxes for the reception of broadcast and broadband digital TV and multimedia applications with a single user interface. Nowadays, consumers have available a wide range of advanced interactive services via their television set, such as Video on Demand (VoD) and catchup TV, as well as internet applications, including video telephony, gaming or shopping. It is reasonable to think that this immense growing content availability should improve the TV entertainment experience by its own, but the lack of personalization services hinders the experience, signaling the need of personalization as the Internet growth signaled the need for searching engines in the last decade.

\footnotetext{
${ }^{1}$ This work has been partially supported by the Community of Madrid (CAM), Spain under the contract number S2009/TIC-1650.

Patricia Arias Cabarcos is with the Telematic Eng. Department, Carlos III University, 28911, Leganés, Madrid, SPAIN (e-mail: ariasp@it.uc3m.es).

Rosa Sánchez Guerrero with the Telematic Eng. Department, Carlos III University, 28911, Leganés, Madrid, SPAIN (e-mail: rmsguerr@it.uc3m.es). Florina Almenárez Mendoza with the Telematic Eng. Department, Carlos III University, 28911, Leganés, Madrid, SPAIN (e-mail: florina@it.uc3m.es). Daniel Díaz-Sánchez is with the Telematic Eng. Department, Carlos III University, 28911, Leganés, Madrid, SPAIN (e-mail: dds@it.uc3m.es).

Andrés Marín López is with the Telematic Eng. Department, Carlos III University, 28911, Leganés, Madrid, SPAIN (e-mail: amarin@it.uc3m.es).
}

Personalization and usability emerge as key features to prevent viewers from feeling overwhelmed by complex management tasks allowing them to squeeze their TVs getting the most from them.

According to these premises, and pursuing to enhance the viewing experience, we introduce in this article a presenceaware personalized TV system. Our contribution is twofold, it provides presence awareness, detecting and identifying which users are present at each moment, and personalization, so it has the ability to show the most appropriate content depending on the users currently viewing the TV.

Related works in the field usually base the personalization on content-filtering based according to recommendation techniques and semantic rules as in [2]. The combination of personalization techniques with presence awareness to automatically generate content is an innovative aspect of our solution. Moreover, we address new challenges such as privacy-based filtering and group preference modeling besides individual preferences.

We envisage future TV systems to be smart enough to determine what a user (or group of users) wants to watch. Therefore, presence detection and individual identification are essential features for providing tailored services.

Presence awareness can be achieved by means without requiring high investments from user side. For instance, user personal devices, as mobile phones, can interact with the personalized TV service in a seamless way to communicate user presence, which is a pivotal concept as envisaged by Weiser in his definition of ubiquitous computing [3].

With the aforementioned goals in mind, we propose FamTV: a prototype architecture for presence-aware personalization aimed on enhancing the TV experience through family-oriented applications.

The rest of this paper is structured as follows: Section II provides a brief background on hybrid TV systems and personalization, identifying the challenges to be faced. Section III provides a global overview of the proposed architecture. Then, Sections IV, V and VI, describes the different layers of FamTV. Section VII describes the prototype implementation. Related work is sketched out in Section VIII. Finally, Section IX summarizes the presented work and some future lines.

\section{BACKGROUND}

The objective of this section is to give an overview of the hybrid TV systems and TV content personalization. 


\section{A. Hybrid TV: main concepts and standardization}

The concept of hybrid television, depicted in Fig. 1, refers to the combination of traditional broadcast services together with external internet contents obtained via a broadband connection. The convergence of these two content sources broadens the possibilities to offer visually-attractive and highly enjoyable content on a TV screen. The sole requirement for and displaying those contents is a hybrid TV or set-top box.

Basically, applications on hybrid devices can be classified into broadcast-related applications, which are associated with broadcast television, radio or data channel, and applications with no relation to any broadcast service, also called broadcast-independent applications. Hence, the services delivered through hybrid TV include $\mathrm{VoD}$, interactive advertising, voting, games, social networking and also program-related services, such as Electronic Program Guides (EPGs). An example of application that merges both worlds is the superposition of widgets of well known social web services (e.g. facebook, twitter) over a broadcasted program to show information related with the content.

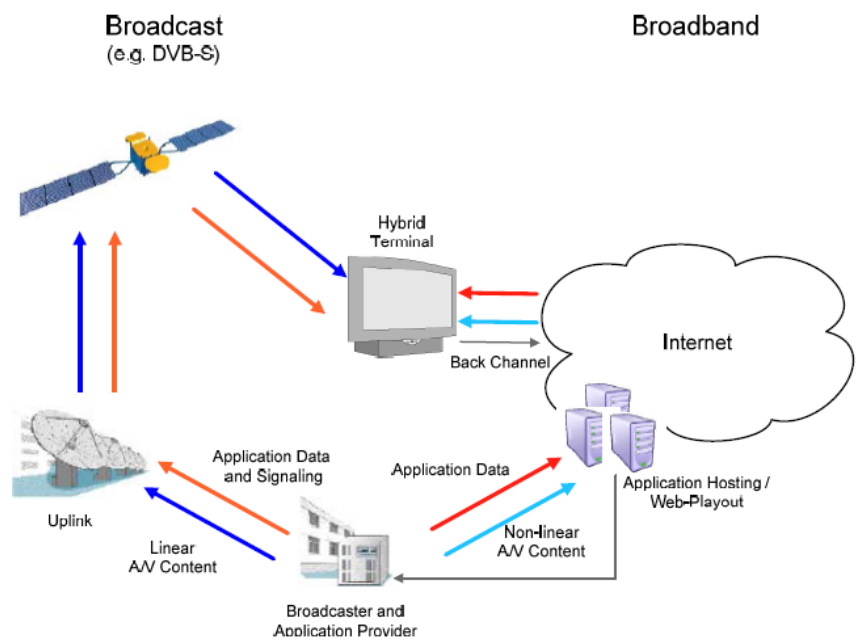

Fig. 1. Hybrid TV system overview extracted from the HbbTV specification [1]. The figure shows contents received from traditional broadcast operators can be coalesced with, for instance, Internet applications.

Despite the concept of hybrid TV is anything but new, the attention has recently increased. This interest is motivated by two fundamental reasons: internet access is almost ubiquitous with higher bandwidths due to new last-mille access technologies, and the richness of current web 2.0 services, which make content merging feasible, as well as appealing.

The most recent standardization activity has been conducted by the HbbTV European initiative. The goal of HbbTV is the definition of an open standardized HTML-based system that allows efficient content development by leveraging existing online services, independently of specific manufacturers or platform operators. The HbbTV specification [1] relies on three existing standards: CE-HTML [4], which profiles the application language (CSS, XHTML, JavaScript); the Open
IPTV Forum's browser profile [5], that provides interfaces to the DVB world; and the DVB's Application Signaling specification [6], which describes transport over broadcast and HTTP. The goal of HbbTV specification is the definition of a specific profile of available technologies, rather than a defining a new one.

As the reader may infer, hybrid TV brings a great potential to interactivity that could not be achieved with nowadays regular TV. However, the coexistence of many content providers results on a overload that hampers TV usage turning simple tasks, as selecting contents to watch, into confusing and difficult. For this reason, personalization is required since offers a customized experience to the TV consumer.

\section{B. Personalization of $T V$ contents}

As we have mentioned before, the explosion in the amount of available TV channels and other contents over hybrid television platforms (broadcast or internet protocols) turns searching and locating interesting content out a cumbersome task for the user. In this context, personalization research is concerned with the adaptation of content (e.g. movies, news, advertisements) in order to show elements and services that are relevant to the viewer and fit her preferences. Filtering systems designed for this purpose are usually classified into two categories according to the underlying recommendation mechanism [7] they use: content-based and collaborative filtering.

Content-based filters contents according to the user history records. Thus, these systems recommend items that are similar to the ones she preferred in the past. Collaborative filtering systems recommend items that people with similar tastes and preferences liked in the past.

Other approaches combining both techniques, as [8] and [9], have been also proposed. But hybrid TV poses new specific challenges in personalization research. For example, web elements that so far were typically viewed on PCs, can now be placed in the TV screen while broadcast content is being displayed. However, there are substantial differences between PC and TV environments. Specifically, we can assert that unlike the computers, TVs are social, and people watch it together. In fact, most of the time there are more than one viewer in front of TV.

This particular condition raises privacy problems: e.g. a user may want some items to be presented only when she is alone in front of the TV but not when the whole family is present. Furthermore, group modeling techniques are desired to offer suited, relevant items for the family members watching TV at a given moment. We aim to capture these new constraints and apply them to content presentation. Thus, we propose personalization framework for hybrid TVs that combines content-based filtering with security and privacy management. Moreover, the contextual information can be enriched with presence data in order to allow user/group detection, providing dynamic adaptation in a seamless and natural manner. 


\section{Presence-Aware TV OVerview ANd ARCHITECTURE}

FamTV is a framework that can be used to build presenceaware personalized applications. In order to show the benefits of FamTV, in this section, we describe some potential use cases that can be for applications using FamTV framework.

\section{A. Use cases}

Switching a TV set on, selecting a channel with the remote and adjust the volume is one of the task any user performs regularly. FamTV provides the means to build an application that shows personalized content immediately after the user switches the TV on. Contents, including several channels in small windows, widgets or preferred news can be automatically displayed according to the preferences and habits of the person who is currently in the room.

FamTV provides also prediction-based services that can be useful in several circumstances. For example, if the viewer is away when her favorite TV show starts, the application will detect it and start recording the show. The content will be ready when the user presence is detected.

Our framework supports exporting profiles between devices. That could make the difference when a user that has been watching TV for a long time in a device, so his profile is very rich and the applications behaves as he likes, is moving for long period of time. FamTV enables a user to move his profile between different devices in order to maintain a smooth personal experience even when changing his usual location.

FamTV protects user privacy. When a viewer is watching $\mathrm{TV}$, her sensitive widgets, such as social network comments, are automatically hidden when the presence of another user is detected.

\section{B. Architecture}

The architecture of FamTV is comprised of a series of interconnected software blocks that distribute all the functionality in a modular fashion. Fig. 2 presents components in a layered model. The Interface Layer at the top of the architecture is the part handling user application interfaces, so the closer to the user. The interface layer is composed by the Personal Content Adapter (PCA) and the Presence Manager Service (PMS).

On the one hand, the Presence Manager Service provides endpoints to detect which users are in the room. Currently, we have only implemented a Bluetooth service since this technology is widely deployed and present in almost every mobile phone today. However, other wireless-based detection techniques could be supported (e.g. Radio Frequency Identification, RFID). We enumerate and discuss different detection techniques in Section IV.

On the other hand, the Personal Content Adapter offers a user-friendly web interface for configuration. As far as configuration concerns, the configuration dashboard contains the following tasks: adding family members, registering devices for detection and adjusting user preferences and security policies. These tasks can be accessed on TV by means of the native browser of the hybrid device, or they can be updated via the Internet.

All the logic functionalities required by the applications in the upper layer are performed by modules in the Logic Layer. Such operations include profile creation and maintenance, user device and user identity mapping, managing of privacy and security features, etc. To dive deeply into the details of these modules, we provide an individual explanation of each one in the following sections.

Finally, the Data Layer accommodates the information required for the applications to operate, that is: user credentials (e.g.: username/passwords), security policies and user/group profiles and history logs. FamTV manages all these data locally so it is impossible for providers to track the user behavior, thus improving privacy.

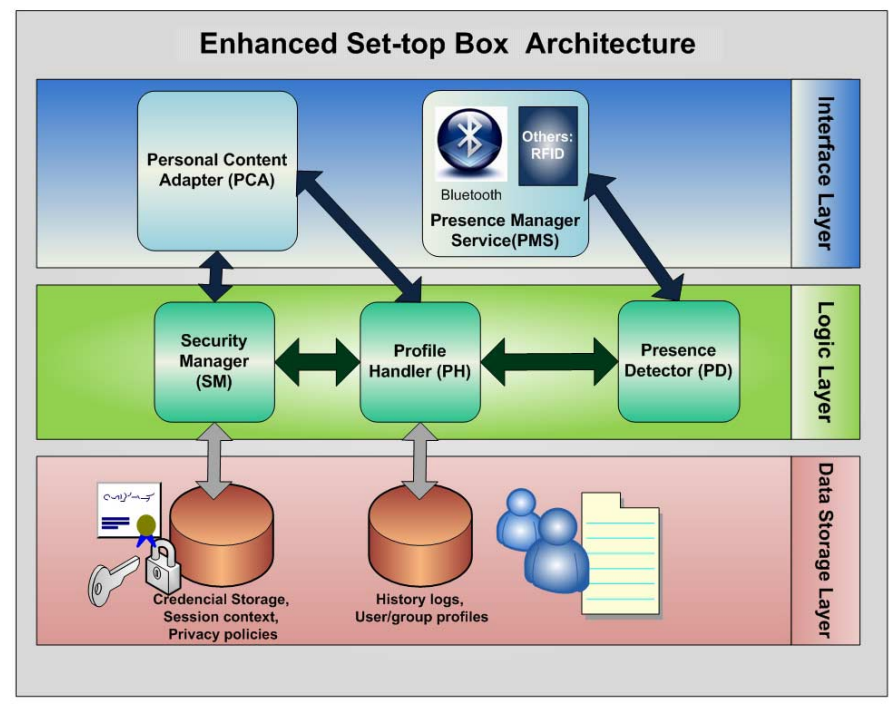

Fig. 2. Architecture for presence-aware personalized TV

\section{INTERFACE LAYER}

FamTV offers an interface layer consisting of two modules: the Personal Content Adapter (PCA) and the Presence Manager Service (PMS). The PCA provides a user-friendly web interface for configuration and includes a main portal to display FamTV applications and to register devices for detection. Regarding the PMS, it adds an interface to our connected Set Top Box in order to perform presence detection.

\section{A. Personal Content Adapter}

The Personal Content Adapter module is in charge of adapting the content according to the current viewer(s). Note that this content adaptation procedure implies adjusting user preferences and security policies. Adaptability can be achieved through either explicit or implicit profile based techniques. In the first case, the system receives information about user's preferences. However, these methods cannot adapt the content dynamically since changes are not detected unless explicitly provided by the user. 
In regard to the implicit profile based techniques, the system can deduce users' preferences by analyzing their viewing history. Our proposal combines both techniques in order to analyze the initial individual or group profile and merge it with the inferred preferences obtained from the viewing history.

On the other hand, the PCA is also responsible for collecting, processing and displaying TV shows, personal widgets, etc, to TV viewers. To carry out these tasks, the Personal Content Adapter module works as follows:

Firstly, the PCA collects information from the broadcast or broadband flows. Then, it applies a content filter and a privacy and security filter that have been composed by the Profile Handler. The technical details about the composition of the filters can be found below in Section V.

Eventually, with the introduction of web technologies and Java code, we achieve automatic web content adaptation. The PCA shows the contents that fit the best to the user or group of users that are present in the room. Also, this module automatically hides any elements considered private when another user comes into the room.

\section{B. Presence Manager Service}

The Presence Manager Service (PMS) provides a web interface for registering users that belong to the family. In addition, the PMS offers a detection interface and notifies Presence Detector events with the aim to maintain the presence context at each moment.

Currently, there are multiple detection techniques that allow us to know which users are in front of the television. We will focus on the following methods: Bluetooth, RFID and Natural Interaction. In this section, we briefly describe them and discuss their advantages and drawbacks to be used in the proposed architecture.

Bluetooth is a wireless protocol for exchanging data over short distances from fixed and mobile devices. Locationaware systems based on Bluetooth technology are low-cost and low-power technology solutions. However, a disadvantage of applying Bluetooth for presence detection in our architecture is that user presence is detected in a range of $10 \mathrm{~m}$ (Bluetooth class 2) and the intended accuracy is around 2 or $3 \mathrm{~m}$.

RFID (Radio Frequency Identification) is a technology that uses radio communication to exchange data between a reader and an electronic tag attached to an object, for the purpose of identification and tracking. The basic method of location using RFID tags is indication of proximity. The primary advantage of an RFID positioning system is that RFIDs are light, small and very cheap. The main disadvantage is the relatively short effect range, typically $1-2 \mathrm{~m}$.

Natural interaction (NI) refers to a concept where humandevice interaction is based on human senses, mostly focused on hearing and vision. Thus, facial and voice recognition techniques can be used for automatic identification of users. Frameworks such as OpenNI [10] provide application programming interfaces (APIs) that allow the development of
NI applications by enabling communication both with hardware sensors and middleware components. The main advantage for identification is that users are not required to carry a personal device.

There are several kinds of benefits in the RFID technology when compared, for instance, with Bluetooth: RFIDs do not need to go through all the steps required when pairing Bluetooth devices; RFID technology does not have the kind of security or power consumption problems that Bluetooth suffers from [11]. On the other hand, innovative frameworks for Natural Interaction, such as OpenNI, have some attractive features to perform presence detection and identification with high precision.

Our prototype implementation uses Bluetooth detection since it was the best solution for a proof of concept, taking into account the easiness of development and its wide adoption. Hence, we assume that FamTV users have a mobile phone with Bluetooth connectivity. Obviously, our approach is not flawless. For instance, it may happen that the presence of a user is not detected because her device is not connected at the moment or that a user in a contiguous room is detected as if she was in front of the TV because she is inside the detection range.

In the future, we aim to complete an enhanced implementation with other detection mechanism that fits better into the seamless philosophy of the architecture (i.e. NI), eliminating the need to carry a personal device. Sensors, like microphones and cameras, are good candidates since 1) they improve the freedom of the user, and 2) their presence in increasingly available in many home entertainment systems like the Kinect for Xbox 360 and others.

\section{LOGIC LAYER}

The core of FamTV is built up of the following logic components:

\section{A. Presence Detector}

The Presence Detector (PD) currently consists of Bluetooth server and client applications for the set-top box (STB) and the mobile phone respectively. Note that the first time that a user registers in the FamTV system, she has to associate her profile with the device that will be used for detection (device pairing).

The PD is in charge of collecting the Bluetooth MAC addresses (or other hardware identifier) of the devices near the TV at every moment. In addition, the PD is connected to the Profile Handler (PH) to perform profile management. Therefore, detection is automatic and if the number of family members varies, the PD notifies this event to the PH which readapts the content dynamically.

The idea is that the TV should be able to automatically recognize a viewer when she approaches the system. Similarly, when the user leaves, the PD should be able to detect this event and to automatically reconfigure its appearance. 


\section{B. Profile Handler}

The Profile Handler (PH) builds an Association Table (AT), so that each Bluetooth MAC address is assigned a user identifier (User ID). When the $\mathrm{PH}$ receives a notification from the PD, it looks up in the AT to retrieve the corresponding user profile and asks the Security Manager to obtain an applicable security policy. Moreover, our proposal also considers the case of detecting the presence of multiple users. So, we introduce a double filtering mechanism based on a set of algorithms for individual and group personalization. Hence, the $\mathrm{PH}$ is able to construct filters in order for the Personal Content Adapter to analyze the multitude of available channels, programs and web items and select the most relevant ones. The complete methodology is explained below in more detail.

The goal of the system is to filter the available items and show only those that are more relevant to the user(s), taking also into account security and privacy.

The item space is composed of every content or application that can be displayed by a hybrid TV (programs, widgets, etc). The user space contains a finite number of elements: the family members. And each element of the user space is defined by a profile that includes various user characteristics, such as age, favorite channels, genre preferences, etc.

We divide this profile information into two subsets: data related to security and privacy configuration; and data for content personalization. The first subset of data is sent to the Security Manager and translated into a security policy. On the other hand, all the information that is relevant for personalization is represented based on Vector Space Model techniques [12]. Thus, when a user registers her profile, an initial Personalization Vector $(P V)$ is created with the form:

$$
P V_{i}=\left[w_{1} w_{2} \ldots w_{n}\right], w_{i} \in\{0,5\}
$$

Where each component $w_{\mathrm{i}}$ represents the degree of preference that the user assigns to attribute $i$. Values of $w_{\mathrm{i}}$ range from 0 to 5 . And the set of attributes (or corpus) is composed of a number of ordered keywords that can be used to categorize items, either these items are programs or web applications. For example, a $P V$ with values [5 4] for the set of attributes (sport, terror) means that the user likes sportrelated items with the maximum degree of preference, and that she likes terror-related items with a degree of preference equal to 4 . So, the system will put sport programs in the first place when showing recommendations for this user.

The initial $P V$ is constructed based on the explicit data provided by the user at registration time. But FamTV is also fed with implicit information inferred from the viewer history. So, we define an updating function to adjust the preference weights in the initial $P V$ as the viewer uses the system:

$$
w_{i}=\alpha \cdot w_{i, \text { previous }}+\beta \cdot U F_{i}
$$

Where $\alpha, \beta<1$ and $(\alpha+\beta)=1$. By varying these two parameters we can assign more importance to the behavior history (i.e. items preferred by the user in the past) or to the new information, respectively. The Updating Factor for attribute $i\left(U F_{i}\right)$ represents the proportion of time a user spends in watching contents that are related to attribute $i$, and this factor is expressed as:

$U F_{i}=\frac{T_{i}}{T_{\text {total }}} \cdot M D P$

Note that the proportion of time in $U F_{i}$ is multiplied by the MDP or Maximum Degree of Preference (i.e. 5) in order to express the result in the scale from 0 to 5 that we defined for the preference metric. Thus, considering $U V_{i}$ as the Updating Vector that contains all the UFs for the set of attributes in the system, Personalization Vectors are updated by calculating:

$P V_{i}=\alpha \cdot P V_{i, \text { previous }}+\beta \cdot U V_{i}$

We take the previous equations as the basis to achieve personalization in the FamTV system.

In order to show personalized content, all the available items are also represented by an Item Vector (IV) whose terms are binary: component $i$ will be 1 if the item can be categorized by attribute $i$, and 0 otherwise. Thus, an ordered set of preferred items is obtained by weighing each Item Vector with the Personalization Vector: the higher is the addition of the weighed terms in the Item Vector, the more relevant is this item for the user.

In the previous example, a sport program would have an $I V$ with components $\left[\begin{array}{ll}1 & 0\end{array}\right]$, whereas a terror film would be represented by $\left[\begin{array}{ll}0 & 1\end{array}\right]$. Thus, after weighing both vectors with $P V=[54]$, the sport program will be recommended first.

Finally, after a set of personalized items is obtained by applying the described algorithm, a new filter is used to discard those contents that must not be shown according to the applicable security policies.

A security policy is defined by a set of rules with the form "if condition then action", but it will be explained in more detail in Section VI.

The case of group personalization is similar to the singleuser case. Group profiles are not required to be created manually. Instead, the information is dynamically acquired by the system. A group profile is automatically created whenever a new group of registered viewers appears in front of the TV. Components in the initial GPV (Group Personalization Vector) are filled by merging individual $P V s$ : degrees of preference are set to the minimum values in the $P V S$ of the users that will form the group. Next, group personalization is achieved by applying the same algorithms described for a single user, but using the $G P V$ for the operations instead of using an individual $P V$. Furthermore, the security filter for a group is based on the most conservative individual policy. 
To summarize, Fig. 3 depicts the double filtering process that is employed to display the final contents, as well as the information required by the system.

The improvement over the traditional information retrieval approaches in TV scenarios comes from the use of detailed profiles that contain information about users' tastes, preferences, and needs. The most significant innovation lies in the technique for group personalization, which provides automatic learning using implicit information extracted from group behavior over time. Moreover, the system is enhanced by applying security and privacy filters, issues that have not been yet considered in related work.

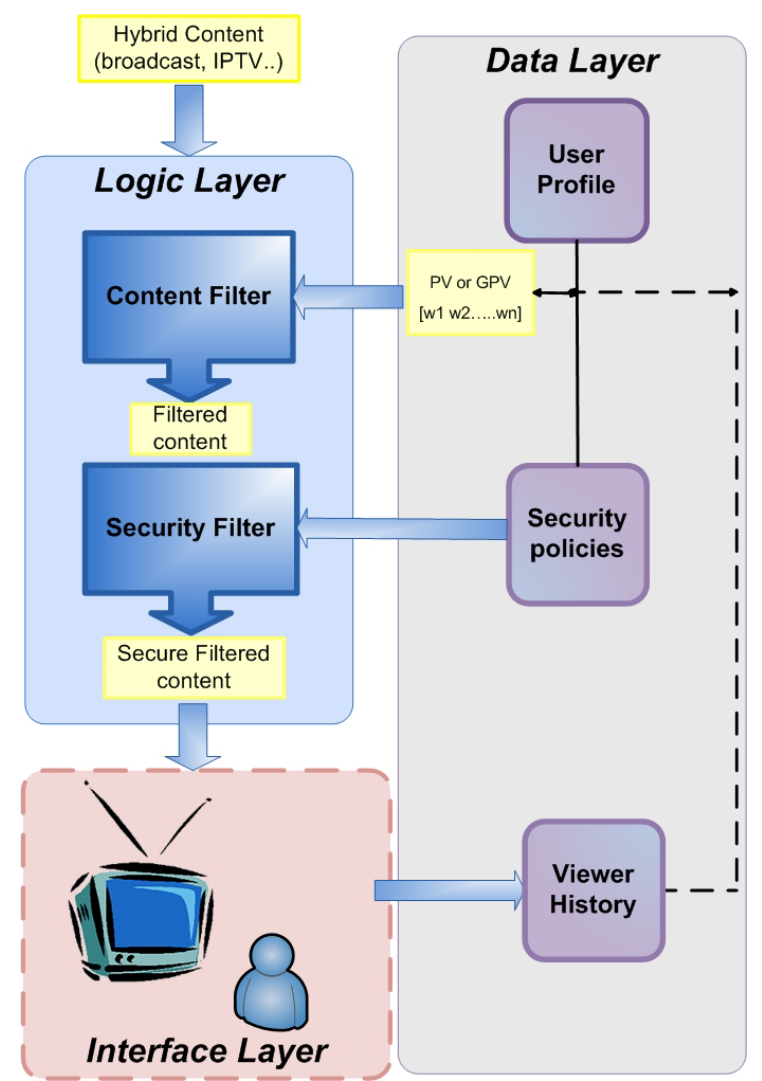

Fig. 3 . Filtering procedure to show personalized contents in FamTV.

\section{Security Manager}

The Security Manager (SM) module is in charge of dealing with security and privacy features. It interacts with the Profile Handler in order to determine the most appropriate security configuration for the current user or group of users. This block has three fundamental functions, namely:

\section{1) Policy enforcement}

Security policies are created and managed by this module to ensure that an adequate security context is active. For instance, parental control rules can be established to prevent children from watching inappropriate contents when their parents are away. Apart from limiting access to ageappropriate content, rules may be defined to set other usage constraints such as, for example, placing time limits.
2) Credential management

User credentials are stored by this module to provide additional functionality in the application. For example, when a specific user is watching TV, her personal widgets could be automatically presented without asking for authentication since the SM performs this task on behalf of the viewer.

\section{3) Session management}

In order to create and maintain a valid security context at each moment, the SM also manages sessions that can dynamically change and readapt depending on the present users.

\section{Data Storage Layer}

The Data Storage Layer accommodates the information required for the system to operate, that is: user/group profiles, user credentials, security policies and history logs.

Users of FamTV must register their profile via a web interface before they start using the system. A user is asked to enter different kinds of information. First, the user provides personal data (age, name) and privacy preferences, which will be used in the security filter. Next, the user selects her preferences regarding contents. For this purpose a list with the main categories in the DVB standard [13] is presented. Every item in the list must be ranked by the user with a degree of preference between 0 and 5. Examples of these categories are: comedy, romance, documentary, variety show, water sport, talk show, news, entertainment, cooking, etc. If a specific category is not ranked, a default value of 2.5 is assigned and the preference will be later derived from the viewer history. As explained before, group profiles are automatically created by the system.

Users must also register a Bluetooth device for presence detection so that the system can build an association between profiles and devices and provide automatic adaptation.

In order to adapt the contents of FamTV to preferences and habits of family members, a history log that records individual and group behavior is maintained. More specifically, the history log contains information about which programs and contents have been consumed by every user or group as well as the initial and final times. This information is the source for the calculation of the Updating Vectors described in the previous section.

Regarding security, every user can store her credentials (e.g. username/passwords, digital certificates) that are required by external applications that can be accessed from the TV: personal widgets, payment services, etc. Thus, the system will act on behalf of the user and perform authentication in the different applications providing an easy and seamless navigation experience.

Finally, security policies are defined by a configurable set of rules that evaluate conditions based on profile attributes in order to deny or grant permissions for a subject to access particular items. Conditions are whether boolean expressions that consider attributes, such as the user age; or rules based on other context variables, such as time. An example of a timebased rule is the following: "John may access TV content from $4 \mathrm{pm}$ until $7 \mathrm{pm} "$. 
It must be noted that all the described data are stored locally in the system, in order to preserve user privacy.

\section{Prototype Implementation}

Our hybrid TV demonstrator is a proof of concept for a PC environment using open source technologies according to the test scenario depicted in Fig. 4.

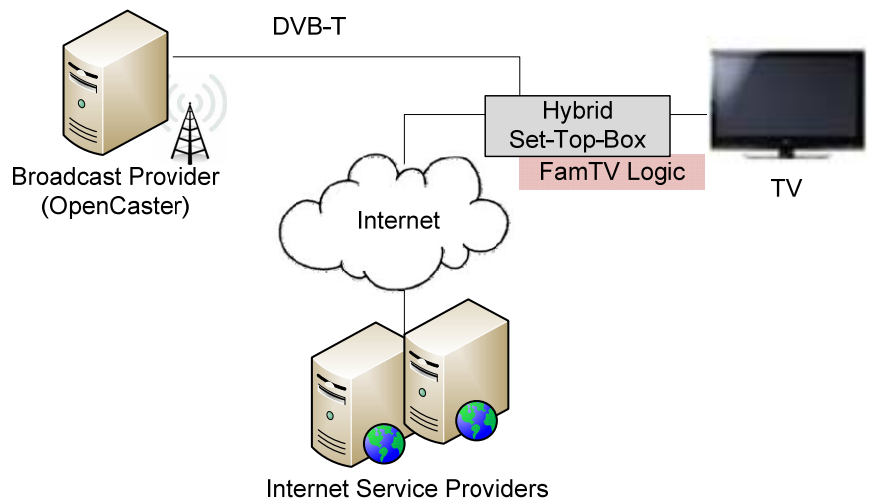

Fig. 4. Test scenario.

For the broadcast part we are using OpenCaster [15], an open source MPEG2 transport stream data generator and packet manipulator, in order to create the broadcast streams. Moreover, this framework allows us to enrich the result with service information as well as with other DVB services.

Regarding the FamTV logic, the web interface for the Personal Content Adapter to register user profiles has been completed. We have also tested the Bluetooth connectivity function in the Presence Detector, as well as the interaction of this module with the Profile Handler. Furthermore, an adaptation service is being developed to offer personalized EPGs based on XMLTV [14] feeds that vary according to which users are detected. For this purpose, the algorithms described in Section V have been developed in Java.

We are improving the Personal Content Adapter, working to integrate the information in the generated broadcast streams with the web EPG to offer a really hybrid experience and apply personalization filters to both kind of contents.

\section{RELATED WORK}

As far as the related work is concerned we found several research initiatives in the field. The main approaches to personalization are: collaborative filtering and content filtering. In this context, typical research directions in TV personalization are related to development of more accurate, effective and efficient algorithms with particular focus on hybrid solutions that combine the basic user modeling and the prediction methods [16][17].

Furthermore, other related works in the field are focused on semantic rules and ontologies [2]. However, current approaches are based on individualized modeling of the viewing experience for each user but do not consider the case of group modeling. Furthermore, none of these works deals with privacy and security issues. Our system allows the establishment of privacy rules and provides security management functions. In addition, the combination of content filtering techniques with presence detection to automatically adapt contents is an innovative aspect.

We aim to address the main limitations regarding TV personalization by defining a user-centric architecture that takes into account group-modeling techniques, since watching television is most a group experience rather than a singleviewer activity [18].

\section{CONCLUSIONS ANd FUtURE WORK}

Presence-aware personalized system combines the benefits of content-filtering and presence detection technologies to achieve seamless personalization. With the introduction of the Presence Manager Service and the Personal Content Adapter, we have designed a user-centric architecture that allows the set-top box to know who is watching TV in order to act accordingly.

Furthermore, security and privacy are also considered. The addition of these features is of special importance: on the one hand, automatic management of user credentials eases the task of authentication with the new applications that can be accessed by hybrid TVs; on the other hand, privacy rules can be established, which is essential having into account that TVs are usually located in a common place at home and most of the time there are more than one viewer.

Usability is paramount in a system like FamTV, and we expect to obtain measures on user acceptance through testing using our proof of concept implementation and evaluation with real users.

As future work, we want to enhance the presence detection module by adding other mechanisms apart from Bluetooth and allow natural interaction with the users. Moreover, it would be interesting to test other prediction algorithms [19] to construct more intelligent services.

Further research is needed to define a delegation model and policies allowing the transfer of tokens and permissions between different users, for example, for payment services.

\section{REFERENCES}

[1] European Telecommunication Standards Institute, "Hybrid Broadcast Broadband TV", ETSI TS 102796 V1.1.1, June 2010.

[2] S.Alam, Z.Iqbal, J.Noll and M.R.Chowdhury, "Semantic Personalization Framework for Connected Set-Top Box Environment", Advances in Human-oriented and Personalized Mechanisms, Technologies, and Services, 2009.

[3] M. Weiser, "The Computer for the 21st Century", Scientific American, September 1991.

[4] Consumer Electronics Association, "A Web-based Protocol and Framework for Remote User Interface on UPnP Networks and the Internet (Web4CE)", CEA-2014-B, June 2006.

[5] Open IPTV Forum, "Release 1 specification, volume 2 (V1.1): Media Formats", Tech. Report, January, 2009.

[6] European Telecommunication Standards Institute, "Digital Video Broadcasting (DVB); Signalling and carriage of interactive applications and services in Hybrid Broadcast/Broadband environments", ETSI TS 102809 (V1.1.1), January, 2010.

[7] G. Adomavicius and A. Tuzhilin, "Toward the Next Generation of Recommender Systems: A Survey of the State-of-the-Art and Possible Extensions", IEEE Transactions on Knowledge and Data Engineering, pp. 734-749, June, 2005. 
[8] P. Cotter and B. Smyth, "A Personalized Television Listing Service",. Communications of ACM, vol. 43 (8), 2000.

[9] A. Martinez, J. Arias, A. Vilas, J. Garcia Duque and M. Lopez Nores, "What's on TV tonight? An efficient and effective personalized recommender system of TV programs", IEEE Transactions on Consumer Electronics, vol.55, no.1, pp.286-294, February 2009.

[10] The OpenNI organization, "OpenNI User Guide", December 2010. Available at http://www.openni.org/.

[11] H. Seppä and J. Vesa, "Wireless Functional Environment: The Future of Wireless Service Delivery", Hong Kong Mobility Roundtable, 2005.

[12] D. L. Lee, H. Chuang and K. E. Seamons, "Document ranking and the vector-space model", IEEE Software, 14(2), pp. 67-75, 1997.

[13] European Telecommunication Standards Institute, "Digital Video Broadcasting (DVB); Specification for Service Information (SI) in DVB systems", ETSI EN 300468 V1.9.1, March 2009.

[14] The XMLTV Project, http://wiki.xmltv.org/.

[15] A. Berger, L. Pallara and R. Schatz, "An opensource framework for DVB-* transmission", Proceedings of the 16th ACM international conference on Multimedia (MM'08), Vancouver, Canada, October 2008.

[16] K. Chorianopoulos. "Personalized and mobile digital TV applications", Multimedia Tools and Applications, volume 36, no. 1-2, pp. 1-10, Springer Netherlands, January 2008.

[17] B. Bezerra, F. Carvalho, G. Ramalho and J. Zucker. "Speeding up Recommendation Systems", Proceedings of the AH2002 Workshop on personalisation in future TV, Malaga, Spain, 2002.

[18] J. Masthoff, "Group modeling: selecting a sequence of television of items to suit viewers", User Modeling and User-Adapted Interaction, vol. 14, no.1, pp.37-85, 2004.

[19] A. Rodriguez-Carrion, C. Garcia-Rubio and C. Campo, "Performance Evaluation of LZ-Based Location Prediction Algorithms in Cellular Networks", Communications Letters IEEE, 14(8), 707 - 709, 2010.

\section{BIOGRAPHIES}

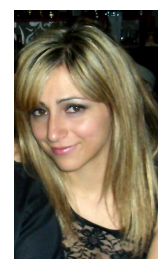

Arias Cabarcos, Patricia received her Telecom. Eng. degree from Univ. Carlos III of Madrid in 2008 and she obtained the MSc degree in Telematics in 2009. Currently, she is pursuing a PhD at the Department of Telematics Engineering in the Univ. Carlos III of Madrid, working within the Pervasive Computing research group. Her research focuses on the problem of identity management in open and dynamic environments, with special attention to risk analysis and the underlying trust models.

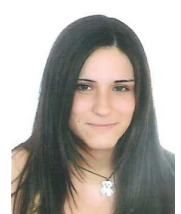

Sánchez Guerrero, Rosa received a Telecom. Eng. degree from Univ. Carlos III de Madrid in 2009. In September 2010 she started her MsC studies in the Univ. Carlos III of Madrid. Currently, she combines her studies with a position as researcher at the Department of Telematics Eng. in the Univ. Carlos III of Madrid, working within the Pervasive Computing research group. Her research topics include the problem of identity management, security and privacy in healthcare.

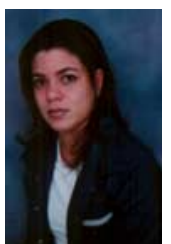

Almenárez Mendoza, Florina (M'07) received the Computer Engineer degree from the University Autónoma of Bucaramanga (Columbia) in 1999, and her Ph.D. degree from the University Carlos III of Madrid (Spain) in 2006. She currently works as an associate professor and researcher in the University Carlos III of Madrid. Her research interests include distributed trust management models for dynamic environments, security architectures in pervasive devices, and security for ad hoc networks.

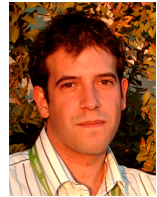

Díaz-Sánchez, Daniel (M’07) received a Telecom. Eng. degree from Univ. Carlos III de Madrid in 2002. He graduated as Master Telematic Engineering (2004) and obtained his PhD (2008) from Univ. Carlos III of Madrid. He works as researcher and teacher at Universidad Carlos III. His research topic is distributed authentication, authorization and content protection activities.

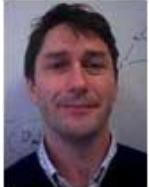

Marín López, Andrés (M’07) received a Telecom. Eng. degree and $\mathrm{PhD}$ from the Technical Univ. of Madrid in 1992 and 1996 respectively. He lectures in Computer Networks and Ubiquitous Computing in the Univ. Carlos III de Madrid, as an associate professor. His research interests include ubiquitous computing: limited devices, trust, security services, and security in NGN. 